\section{Facharztprüfung zur Erlangung des Facharzttitels FMH für Angiologie}

Aufgrund des Weiterbildungsprogrammes, welches am 1. Januar 2002 in Kraft gesetzt wurde, ist die Teilnahme an der Facharztprüfung für diejenigen Kandidatinnen und Kandidaten Voraussetzung für die Erlangung des Facharzttitels Angiologie, welche ihre Weiterbildung in Angiologie bis am 30. Juni 1998 nicht abgeschlossen haben. Es empfiehlt sich, die Facharztprüfung frühestens im letzten Jahr der reglementarischen Weiterbildung abzulegen (Art. 23 WBO).
Erster Teil (schriftliche Prüfung)

Samstag, 26. Oktober 2002, 9.00 Uhr, anlässlich des Unionskongresses in Flims.

Zweiter Teil (mündliche und praktische Prüfung) Donnerstag, 28. November 2002, Inselspital, Bern.

Prüfungsgebühr: Die Fachgesellschaft erhebt bei Anmeldung der Kandidaten zur mündlichen Prüfung eine Prüfungsgebühr von Fr. 1200.-, welche auch die Teilnahme an der schriftlichen Prüfung beinhaltet.

Anmeldung: Inselspital Bern, Angiologische Abteilung, Frau Catherine Gut, 3010 Bern, Tel. 031632 30 34, Fax 03163247 93, E-Mail: catherine.gut@ insel.ch.

Anmeldefrist: 28. September 2002

\section{Facharztprüfung zur Erlangung des Facharzttitels FMH für Rheumatologie}

Aufgrund des Weiterbildungsprogrammes und des Beschlusses des Zentralvorstandes der FMH vom 28. Dezember 2000 ist das Bestehen der Facharztprüfung für alle Kandidatinnen und Kandidaten, welche ihre Weiterbildung nach dem 31. Dezember 2002 abschliessen, Voraussetzung zur Erlangung des Facharzttitels FMH für Rheumatologie. Näheres zu den Übergangsbestimmungen und Ausnahmen ist in der Schweizerischen Ärztezeitung Nr. 6 vom 7. Februar 2001 publiziert.
Mündliche Prüfung

Ort: Rheumaklinik, Universitätsspital Zürich

Datum: Donnerstag, 22. August 2002

Prüfungsgebühr: Die Fachgesellschaft erhebt für die mündliche Prüfung eine Prüfungsgebühr von Fr. 700.-.

Zulassungsbedingung: absolvierte 1. Teilprüfung (MC-Fragen)

Schriftliche Anmeldung: Iris Zehnder, Sekretariat SGR, c/o Schweizerische Rheumaliga, Renggerstrasse 71, Postfach, 8038 Zürich, Tel. 0148740 00, Fax 0148740 19, E-Mail: srl@srl.ch

Anmeldefrist: 30. Mai 2002 


\section{Examen de spécialiste en vue de l'obtention du titre de spécialiste FMH en angiologie}

Conformément au programme de formation postgraduée entré en vigueur le $1^{\mathrm{er}}$ janvier 2002, la participation à l'examen de spécialiste est une condition requise pour les candidats à l'obtention du titre de spécialiste FMH en angiologie qui n'ayant pas terminé leur formation postgraduée en angiologie jusqu'au 30 juin 1998. Il leur est recommandé de passer l'examen de spécialiste au plus tôt durant la dernière année de leur formation postgraduée réglementaire (art. $23 \mathrm{RFP}$ ).
Première partie (examen écrit)

Samedi, le 26 octobre 2002, 9.00 heures, lors du Congrès de l'Union à Flims.

Deuxième partie (examen oral et pratique) Jeudi, le 28 novembre 2002, à l'Hôpital de l'Ile, Berne.

Taxe d'examen: La société de discipline médicale prélève lors de l'inscription des candidats pour l'examen oral une participation aux frais de Fr. 1200.- (y compris la taxe pour la participation au examen écrit).

Inscription: Hôpital de l'Ile, Division d'Angiologie, Mme Catherine Gut, 3010 Berne, tél. 03163230 34, fax 03163247 93, e-mail: catherine.gut@insel.ch.

Délai d'inscription: 28 septembre 2002

\section{Examen de spécialiste en vue de l'obtention du titre de spécialiste FMH en rhumatologie}

Conformément au programme de formation postgraduée et à la décision du Comité central de la FMH du 28 décembre 2000, la réussite de l'examen de spécialiste est une condition requise pour les candidats au titre FMH en rhumatologie qui termineront leur formation postgraduée après le 31 décembre 2002. Pour de plus amples renseignements concernant les dispositions transitoires et les dérogations, veuillez vous référer à la publication du Bulletin des médecins suisses no 6, du 7 février 2001.

\section{Examen oral}

Lieu: Clinique de rhumatologie, Hôpital universitaire, Zurich

Date: Jeudi, 22 août 2002

Taxe d'examen: La Société de discipline médicale prélève pour l'examen écrit une participation aux frais de Fr. 700.--

Conditions d'admission: avoir passé l'examen écrit (QCM).

Inscriptions (par écrit): Iris Zehnder, Secrétariat SSR, c/o Ligue suisse contre le rhumatisme, Renggerstrasse 71, Case postale, 8038 Zurich, tél. 01 48740 00, fax 0148740 19, e-mail: srl@srl.ch

Délai d'inscription: le 30 mai 2002 